\title{
Aging Blepharoplasty
}

\section{Inchang Cho}

Bio Plastic Surgery Clinic, Seoul, Korea

In performing upper blepharoplasty in the elderly, looking younger and keeping the eyelids harmonious with the rest of the face have to be achieved at the same time. The most important goal in upper blepharoplasty for aging is correcting the drooping upper eyelid skin, and in this process, the surgeon may or may not create a double eyelid fold. The pros and cons have to be fully discussed with the patient, but the author personally prefers creating a double fold unless the patient refuses, because it is efficient in correcting and preventing further drooping of the skin. In most patients, the brow is elevated to compensate for the drooping eyelid, and when the drooping is corrected, brow ptosis may ensue. The surgeon has to prepare for these consequences before performing the procedure, and estimate the exact amount of skin to be excised. In the elderly, the skin and the orbicularis oculi muscle is thin, with a decreased amount of subcutaneous fat and retro-orbicularis oculi fat, and in most cases, excision of the skin alone is enough to correct the deformity. Removing large portions of soft tissue may also prolong the recovery period. Unlike younger patients, the lower skin flap should not be stretched too much in the elderly, as it may create an aggressive looking appearance. A few wrinkles in the lower flap should remain untouched to create a natural look. In this article, the author's own methods of performing an aging blepharoplasty are described specifically, with a step-by-step guide and surgical tips.

\section{Keywords Blepharoplasty / Aging / Convalescence}

Received: 22 Jul 2013 • Revised: 1 Aug 2013 • Accepted: 2 Aug 2013

pISSN: 2234-6163 • elSSN: 2234-6171 • http://dx.doi.org/10.5999/aps.2013.40.5.486• Arch Plast Surg 2013;40:486-491
Correspondence: Inchang Cho Bio Plastic Surgery Clinic, Tongyeong Bld. 10th Fl. 405, Gangnam-daero, Seocho-gu, Seoul 137-856, Korea Tel: $+82-2-535-9000$

Fax: +82-2-533-8128

E-mail: bandoeye@hotmail.com

No potential conflict of interest relevant to this article was reported.

\section{INTRODUCTION}

Aging is not avoidable and happens naturally as time passes, but may have a negative effect on social relationships. From this perspective, rejuvenation surgery may bring happiness to people by increasing their confidence and thus helping them to perform better socially. Blepharoplasty of the young focuses on producing an ideal eyelid shape, but in the elderly, looking younger and while at the same time maintaining eyelids that are harmonious with the rest of the face are important factors to take into account. In blepharoplasty for the aging, the most important objective is to correct the sagging of the upper eyelid skin, and in East Asians, this may be achieved by excision of the redundant skin either with or without creating a double eyelid fold. In the elderly, the skin is usually thin, and subcutaneous fat and retro-orbicularis oculi fat (ROOF) are sparse, so minimal excision of the soft tissues should be performed $[1,2]$. This also has an effect on the recovery period, which should be kept as short as possible to allow for rapid adjustment. This article was written to aid young surgeons in their practice, presenting the methods the author uses to perform blepharoplasty for aging patients with the above mentioned factors in mind. 


\section{SURGICAL PROCEDURES}

\section{Design of the skin excision line}

To design the skin excision line in blepharoplasty for aging patients, the upper and lower incision lines, and the medial and lateral margins of the skin being excised, should be determined. The lower line is drawn first, as this is the line where the tarsal plate is fixed to create a double fold. While the patient is in the sitting position, the assistant lifts the eyebrows to expand the upper eyelid skin to $80 \%$ to $90 \%$ of its full length. The surgeon creates several kinds of double fold lines with a probe and shows them to the patient with a mirror so that the patient may choose a line of his/her preference. If the patient already has a double eyelid fold, then the surgeon must determine whether to maintain that line or to create a new line, based on a consultation with the patient using the above mentioned method.

When the lower line is decided, the length from the lower margin of the upper eyelid to the lower line is measured. Then

\section{Fig. 1. Design of the excision lines}

Design of the upper and lower skin excision lines. (A) While an assistant lifts the brow so that the upper eyelid skin is expanded to $80 \%$ to $90 \%$ of its full length (black arrow), the desired double fold line is drawn, which is the lower skin excision line. (B) The line is drawn at the same level after the lifted brow is released, which is the upper skin excision line. The length from the upper eyelid margin to the lower incision line while the brow is lifted, and to the upper incision line while the brow is released, is the same $\left(a=a^{\prime}\right)$ (red arrow). (Reproduced from Cho I. The art of blepharoplasty. Seoul: Koonja; 2013 [3]).
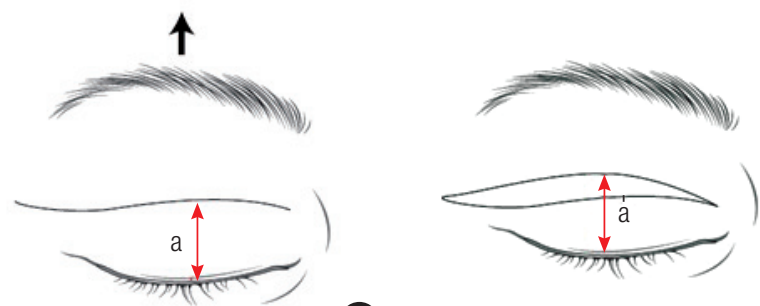

A the assistant releases the eyebrow to its natural position, and the same length from the lower margin of the upper eyelid to the upper eyelid skin is measured and marked. This is the upper border of the skin excision (Fig. 1). As the lower excision line creates a gentle curve, the upper excision line is marked at three points; the level of the medial limbus, the level of the midpupil, and the level of the lateral canthus, and the three points are connected to create the upper excision line with a gentle curve as well.

The medial margin of the designed excision line should never cross the transition area from the upper eyelid skin to the nasal skin. It is also recommended that the lateral margin of the excision line not extend more than $2 \mathrm{~cm}$ beyond the orbital rim. If these lines are crossed, conspicuous scars may form. Decision making in designing the medial portion of the excision line depends on the amount of drooping. Usually, drooping is more severe in the lateral eyelid skin, and brow ptosis is also more frequent at the lateral portion. If drooping on the medial side is minimal, a narrow wedge-shaped excision should be performed as in the illustration (Fig. 2A). If there is moderate drooping, the excision line should extend more medially to excise a greater portion of the skin (Fig. 2B). If there is severe drooping, with wrinkles on the medial eyelid skin and even on the nasal skin, a back-cut should be designed bending upwards as shown in the illustration (Fig. 2C). The width of the back-cut triangle should be designed appropriately as not to create too much tension, which may lead to the creation of scar bands or hypertrophic scars. The excision line in the lateral region should bend upwards about 15 degrees from the gentle downward curve, to follow the crow's feet lines. The turning point is usually 4 to $6 \mathrm{~mm}$ lateral to the lateral canthus, and should be designed with a shorter length in men or patients who want a weak line.

\section{Excision of soft tissue}

After the skin excision line is designed, the skin is excised under local anesthesia. In excising the underlying orbicularis oculi

\section{Fig. 2. Different designs in the medial region}

The design of the skin excision differs depending on the amount of sagging of the medial upper eyelid skin (red arrow). (A) When there is minimal sagging of the medial skin, a narrow wedge-shaped excision is performed. (B) When there is moderate sagging, the excision is extended to the medial side. (C) When there is severe sagging, a triangular backcut is designed. If the width $A$ is too long, hypertrophic scarring and an epicanthal fold-like band may form. (Reproduced from Cho I. The art of blepharoplasty. Seoul: Koonja; 2013 [3]).
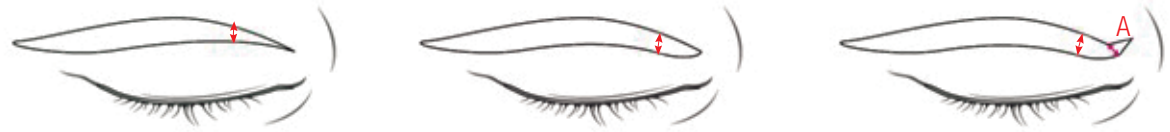

A

B 
muscle, a 1- to 2-mm cuff of muscle from the skin excision line is saved at the upper border to prevent a triple fold, and also at the lower border to prevent a depressed scar (Fig. 3). When excision of extensive amounts of skin is necessary, skin excision may be reduced to prevent brow ptosis by increasing the excision of the orbicularis oculi muscle. The skin and the orbicularis oculi muscle are considered to be one unit, and one may be sacrificed to save the other. In patients with attenuated skin and a sunken appearance, redundant skin is excised but the orbicularis oculi muscle is not excised, to create pretarsal fullness and a youthful appearance.

In case the eyelid is fatty and bulging, a small incision on the septum is created to remove some fat tissue from the lateral compartment. An incision on the septum should be made with caution, as it is a gliding membrane, to prevent adhesion. If there is bulging on the medial side of the upper eyelid, fat removal should be carried out on the medial side as well. The fat on the medial side is denser with thick vessels, so hemostasis must be performed thoroughly. Excision of the retroorbicularis oculi fat should be avoided whenever possible, as this fat aids in the gliding of the orbicularis oculi muscle at the orbital rim. At the lateral portions it is relatively safe to remove small amounts in case

\section{Fig. 3. Muscle preservation during the excision}

When excising the orbicularis oculi muscle (00M) with the skin, a 1- to 2-mm strip of muscle is saved on the upper side (red line). This prevents the formation of a triple fold. On the lower side, another 1 - to $2-\mathrm{mm}$ strip of muscle is saved (blue line) to prevent a depressed scar. (Reproduced from Cho I. The art of blepharoplasty. Seoul: Koonja; 2013 [3]).

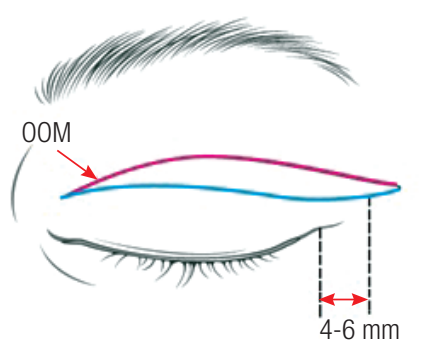

\section{Fig. 4. Excision of lateral portions}

(A) If too much soft tissue is removed from the lateral portion marked 'a' where there is no double fold line, the patient may look cachexic. (B) Because of too much soft tissue removal from the lateral portion, the lateral roundness has disappeared, creating an awkward appearance. (Reproduced from Cho I. The art of blepharoplasty. Seoul: Koonja; 2013 [3]).

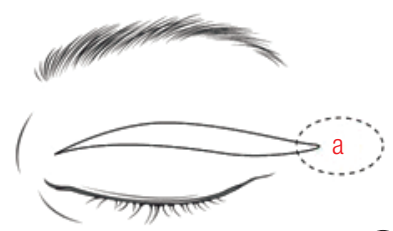

A

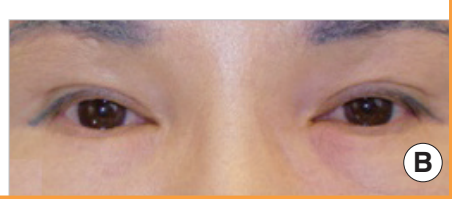

there is too much fullness. If too much of this fat is removed or if the fat close to the periosteum is removed, it may cause adhesions and create depressions, and it may even cause ptosis due to limitation of movement of the eyelids (Fig. 4).

\section{Fixation}

To create a natural double eyelid fold, fixation should be done to the tarsus or the levator aponeurosis at the level where the skin is slightly stretched. If the fixation is done at a higher point, a high and deep fold may be created, ectropion may be induced, and sometimes the tone of the skin becomes darker due to visible small vessels. The author performs the fixation at a level where the skin is stretched to a degree such that only mild wrinkles are left on the lower flap skin. The needle is passed shallowly through the tarsus at this level, then the levator aponeurosis is passed through at a higher level, and finally the pretarsal orbicularis oculi muscle of the inferior flap is passed through and the suture is tied.

Fixation is done at 4 to 5 points, at a relatively inferior level compared to younger patients. The fixation is performed at a higher level than the incision line as it goes medially, and at a lower level as it goes laterally, to create an equal depth of the fold. The most lateral fixation point is usually 4 to $6 \mathrm{~mm}$ laterally to the lateral end of the palpebral fissure, and as there is no tarsus in this region, fixation is made to the levator aponeurosis or the orbital septum (Fig. 5). However, as the levator aponeurosis runs deeper in this region, the double fold may become deeper and abruptly end at this point (Fig. 6). There are three methods to prevent this from happening: 1) A relatively easy method is to fix the levator aponeurosis, the orbital septum, and retroseptal fat all at once. Using this method, the levator aponeurosis aids in creating a stable fixation, while the orbital septum and retroseptal fat prevents deep fixation. 2) Another option is to find a spot where the levator aponeurosis is not too deep and fix to that

\section{Fig. 5. The fixation points in relation to the incision}

Relation of the incision line and the fixation points. The fixation points (red X) move inferiorly in relation to the incision line as it goes laterally, to create a uniform depth of the supratarsal fold. At the most lateral point, fixation is performed to the levator aponeurosis or the septum, as there is no tarsus. (Reproduced from Cho I. The art of blepharoplasty. Seoul: Koonja; 2013 [3]). 


\section{Fig. 6. Relationship of the levator aponeurosis and tarsus}

(A) Lateral to the palpebral fissure, there is no tarsus in the upper eyelids. (B) The double fold line is aesthetic when it extends 4 to $6 \mathrm{~mm}$ lateral to the canthal angle. (C) The levator aponeurosis is located relatively deeper toward the eyelids at the level of the lateral canthus. (Reproduced from Cho I. The art of blepharoplasty. Seoul: Koonja; 2013 [3]).
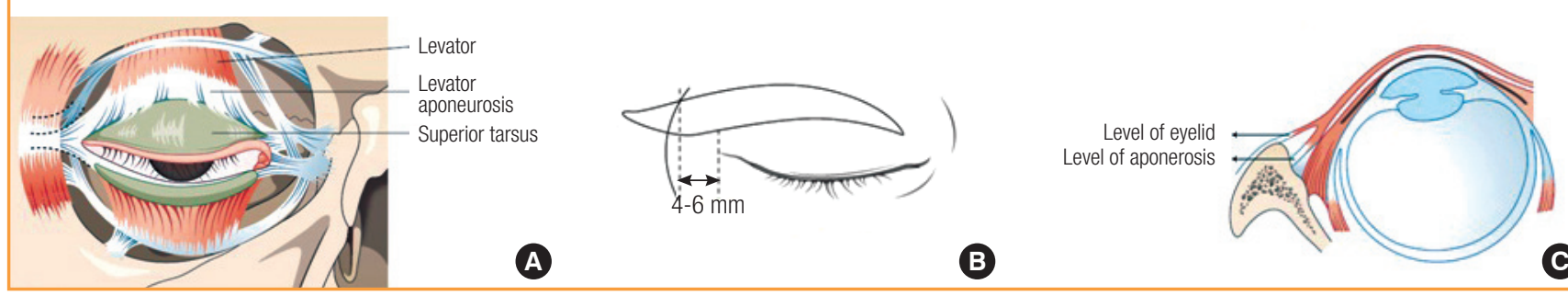

\section{Fig. 7. The direction of fixation sutures}

The direction of fixation sutures. The fixation should be performed radially, with the lateral tarsus to the medial portion of the lower flap, and the medial tarsus to the lateral portion of the lower flap (black arrows). (Reproduced from Cho I. The art of blepharoplasty. Seoul: Koonja; 2013 [3]).

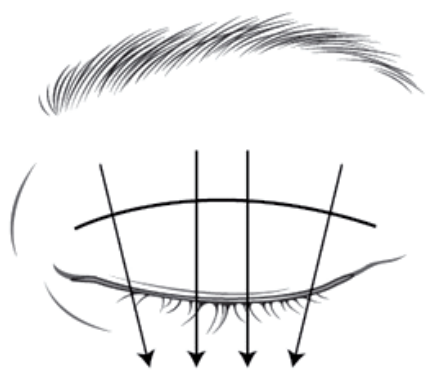

point. 3) Finally, it is possible to dissect the levator aponeurosis to create an aponeurotic flap, and fix that to the orbicularis oculi muscle of the lower flap. When fixation is performed on the orbital septum, the septum close to the levator aponeurosis should be used to create a gentle fold.

The direction of the fixation should be radial, not vertical. The eyelid is not a plane, but wraps around the globe, and as the eyelid moves upwards, the levator aponeurosis fans out radially. Therefore, in performing medial fixations, the suture should be performed between a more medial tarsus and a more lateral orbicularis oculi muscle of the lower flap, and in performing lateral fixations, the suture should be performed between a more lateral tarsus and a more medial orbicularis oculi muscle (Fig. 7).

\section{Skin sutures}

Guiding fixation sutures should be placed while suturing the skin to decrease the tension on the permanent fixation sutures due to swelling. Usually 3 to 4 points of guiding fixation sutures are placed. These sutures are done by passing the needle through the skin and orbicularis oculi muscle of the lower flap, tarsus, and then the skin and orbicularis oculi muscle of the upper flap (Fig. 8), in order to prevent depression scars. Then the rest of the skin is sutured using continuous sutures. Skin sutures, unlike permanent fixation sutures, should be done nearly vertically, not taking into account the length discrepancy between the upper

B

\section{Fig. 8. Guiding fixation sutures}

Guiding fixation sutures. When performing the skin closure, 4-5 temporary sutures are placed as guiding fixation sutures, and removed on postoperative days 3 to 4. (Reproduced from Cho I. The art of blepharoplasty. Seoul: Koonja; 2013 [3]).

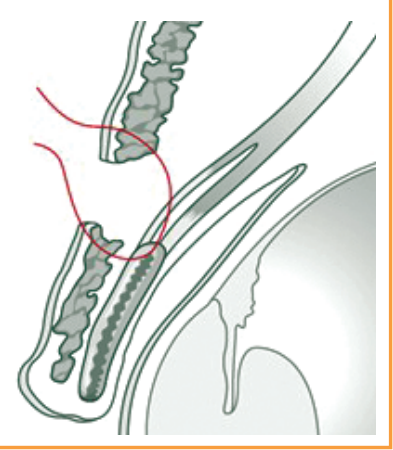

and lower incision lines. Normally, no undermining is necessary when suturing the eyelid, but some undermining at the lateral portion may enable relief of tension on the suture site and ease skin eversion on suturing. After the stitches are removed, taping is done on the lateral portion for 2 weeks.

\section{DISCUSSION}

There are many different ways of performing upper blepharoplasties in elderly East Asians, and the above mentioned procedures are the author's preferred methods. When designing the lower incision line, the brow is lifted by the assistant to minimize the confounding effect of brow soft tissues on upper eyelid sagging [4]. As mentioned above, the upper eyelid skin should be expanded to $80 \%$ to $90 \%$ of its full length. If it is spread out too much, the double eyelid fold will have a higher location and therefore have an unnatural appearance. In addition, to design the precise amount of skin to be excised, the patient must be emotionally stable and close his/her eyes completely during the design. The assistant may slightly press the eyebrow down when drawing the upper excision line.

The skin and the orbicularis oculi muscle should be thought of as one unit. When too much skin has to be excised to achieve the desired result, which may cause brow ptosis, the underlying orbicularis oculi muscle and soft tissue may be excised instead 
to save more skin. But in the elderly there is a deficit of soft tissue volume, so excision of the orbicularis oculi muscle should be avoided whenever possible, as it may induce triple folds. There are reports in which the orbicularis oculi retains its volume and function despite aging and excision of too much tissue will aggravate sunken deformities [5-7]. The author does not excise any orbicularis oculi muscle in more than half of the cases. Saving the orbicularis oculi muscle may also aid in preventing depressed scars. If the lower flap is thin, no dissection of the lower flap is performed to prevent formation of adhesions, which may lead to accordion-like deformities, which form due to narrowing of the skin when opening the eyes. Too much excision of muscle may also lead to darkening of the skin and development of telangiectasia.

Even in patients with a sunken appearance and thin skin, the medial fat may bulge out. This is because the attenuated medial horn allows more fat to protrude out to the medial side [8]. This fat can be removed via a transconjunctival route without damaging the medial horn. The removed fat can be free grafted to correct other depressed areas.

In the elderly, stretched skin due to blepharoplasty creates a strong impression, unlike in young patients, which may seem unnatural. Also, creating a double fold may aggravate this unnatural appearance, and some surgeons are reluctant to perform this procedure in the elderly. However, if the fixation point is determined appropriately with adequate stretching of the lower flap, a natural and aesthetic appearance may be achieved in the elderly (Fig. 8). This is made possible by fully understanding the nature of performing upper blepharoplasties in East Asians.

However, before creating a supratarsal crease, various factors have to be taken into account in elderly patients. Although creating a double fold can have many advantages such as relieving the sagging of upper eyelids and preventing entropion, since the patient has lived without a double eyelid fold for a very long time, there might be some trouble adapting to having a double fold. Also, the recovery period is longer for patients who undergo creation of a double fold. These factors should be fully discussed with the patient before performing the surgery. The bright side is that for the elderly, the supratarsal crease is not fixed as deeply as those performed in young patients. In young patients, the fixation is a bit overdone in case the double fold comes undone, but in the elderly, this is done more weakly so the recovery period is shorter and the fold becomes natural more quickly.

When performing fixation sutures, the lateral points must be treated with care, as there is no tarsus to which to fix the sutures. When the fixation is performed to the septum, it is important to place the suture at a point near the levator aponeurosis. If the point is too far from the levator aponeurosis, the double fold line may be too thin. The length from the canthal angle to the orbital rim is $5.4 \pm 1.5 \mathrm{~mm}$, so shorter patients may have less than $4 \mathrm{~mm}$ of length [9]. In these cases, a septal turnover flap may be made from the septum attached to the levator aponeurosis to be fixed to the double fold line, or even the levator aponeurosis itself may be extended to create a flap to be fixed to the fold line. The direction of fixation of the sutures is also important, and if it is not done radially, oblique wrinkles may form on the medial side of the fold line and the line may end abruptly on the lateral side.

\section{CONCLUSIONS}

In performing blepharoplasty in aging patients, the surgeon should perform the procedure as atraumatically as possible with a minimal amount of tissue manipulation, so that the immediate postoperative results are similar to the long-term results. The result should be mildly ectropic to compensate for the loosening of the double fold line. To shorten the recovery period, performing a skin excisional procedure with internal fixation is preferred. After excision of the skin, the orbicularis oculi muscle should be excised in patients with thick upper eyelids, and it should be preserved by only performing an incision in patients with thin eyelids. The pretarsal tissue should not be removed. Only 3 to 4 slit incisions should be made on the pretarsal tissue, and the orbicularis oculi muscle should be fixed to the tarsal plate through these incisions. The height of the fixation should be at the point where the lower flap skin is stretched $80 \%$ to $90 \%$ of its full length. When performing the fixation, the needle should be passed through the tarsus at a partial thickness, then the levator aponeurosis should be passed through, and finally the orbicularis oculi muscle before the suture is tied. This method shortens the recovery period because blood flow to the orbicularis oculi muscle layer is well maintained, as only slit incisions are made instead of removing the pretarsal tissue. Because the fixation is performed to a relatively low portion of the tarsus and levator aponeurosis, the skin is not overly stretched and the recovery time is reduced, as it does not take long for the line to become natural.

\section{REFERENCES}

1. Hsu AK, Jen A. Estimation of skin removal in aging Asian blepharoplasty. Laryngoscope 2012;122:762-6.

2. Lee JW, Baker SR. Esthetic enhancements in upper blepharoplasty. Clin Plast Surg 2013;40:139-46.

3. Cho I. The art of blepharoplasty. Seoul: Koonja; 2013.

4. Pepper JP, Moyer JS. Upper blepharoplasty: the aesthetic ideal. Clin Plast Surg 2013;40:133-8. 
5. Tonnard PL, Verpaele AM, Zeltzer AA. Augmentation blepharoplasty: a review of 500 consecutive patients. Aesthet Surg J 2013;33:341-52.

6. Lee H, Park M, Lee J, et al. Histopathologic findings of the orbicularis oculi in upper eyelid aging: total or minimal excision of orbicularis oculi in upper blepharoplasty. Arch Facial Plast Surg 2012;14:253-7.

7. Hwang K, Kim DJ, Kim SK. Does the upper eyelid skin be- come thinner with age? J Craniofac Surg 2006;17:474-6.

8. Ettl A, Priglinger S, Kramer J, et al. Functional anatomy of the levator palpebrae superioris muscle and its connective tissue system. Br J Ophthalmol 1996;80:702-7.

9. Hwang K, Nam YS, Kim DJ, et al. Anatomic study of the lateral palpebral raphe and lateral palpebral ligament. Ann Plast Surg 2009;62:232-6. 